\title{
Misconcepts in Management of Severe Malaria
}

\author{
Polrat Wilairatana $^{1 *}$, Noppadon Tangpukdee ${ }^{1}$ and Srivicha Krudsood ${ }^{2}$
}

${ }^{1}$ Faculty of Tropical Medicine, Departments of Clinical Tropical Medicine, Mahidol University, Thailand

${ }^{2}$ Faculty of Tropical Medicine, Tropical Hygiene, Mahidol University, Thailand

\begin{abstract}
Early diagnosis and proper management is very important in management of severe malaria. Although there were many update information's regarding to management of severe malaria, some misconcepts of the clinicians in management remained and should be reconsidered and corrected to avoid harmful management to the patients.
\end{abstract}

\section{Keywords: Severe malaria; Misconcepts}

Malaria is the most important parasitic infection in the world since it causes high mortality. Nearly one million people died due to malaria each year and most of them were children in African countries. No other parasite can cause high fatal patients like malaria. In management of severe malaria, early diagnosis and properly appropriate management of patients is most important and essential. Although World Health Organization (WHO) has worldwide disseminated informative books and handbook regarding management of malaria including pathophysiology, common errors in diagnosis and managements, management of complications [1-4], some misconcepts in management of severe malaria have been found in many hospitals in the world. Mortality rates of severe malaria in many hospitals varied due to many factors and one factor is misconcept of clinicians. Although many hospitals have well-equipments for supportive care of malaria complications such as dialysis facility for acute renal failure and volume ventilators for adult respiratory distress syndrome (ARDS), misconcepts in management of severe malaria may contribute to higher mortality rate of the patients. We would like to comment some misconcepts in management of severe malaria found in one western country. The misconcepts were published in one journal regarding to intensive care [5] as an example as followings:

Neurological Manifestations [5]: The authors mentioned "Computed tomography (CT) scan of the brain may show cerebral edema". There were earlier discrepant studies. Some studies mentioned CT [6] and even magnetic resonance imaging (MRI) [7] of non-fatal cerebral malaria showed no cerebral edema. Another studies of CT [8] and MRI [9] showed cerebral edema. In fatal cerebral malaria patients, mildly swollen brain is commonly found [10]. Cerebral edema may be also found in post-mortem of other non-malarial diseases too. WHO (2010) mentioned no significant difference in mortality between dexamethasone used in cerebral malaria, but gastrointestinal bleeding and seizure were more common with dexamethasone $[2,11]$ and dexamethasone is not recommended as adjunctive treatment in cerebral malaria at presence. However the trails were small and there was no reported effect on disabilitiy [2].

Antimalarial Therapy: Quinine Dihydrochloride and Quinidine Gluconate [5]: The authors mentioned "Intravenous therapy with quinine or quinidine should be switched to oral therapy as soon as the patient is able to take oral medications. It is prudent to administer at least 5 to 7 days of quinine". The present update of severe malaria by WHO [2] in 2010 recommended that parenteral quinine should be given to treat severe malaria patients for a minimum of 24 hours, once started (irrespective of the patient's ability to tolerate oral medication earlier), and thereafter, complete treatment by given an oral course of quinine plus clindamycin or doxycycline or artemisin combination therapy. It is correct that total quinine should be given for 7 days. Shorter courses of quinine (e.g., 5 day-courses) are less effective [10]. In areas of multidrug resistant malaria, quinine should be combined with clindamycin or doxycycline if there is no parenteral artesunate available. Intravenous artesunate was proved to be better than intravenous quinine in treatment of severe malaria in both adults and children $[12,13]$ since artesunate reduced mortality rates more than quinine. Quinidine is recommended outside malaria endemic area where there may be delays in obtaining other antimalarial drugs for severe malaria (e.g. artesunate or quinine). However quinidine should be given by careful rate-controlled intravenous infusion with continuous electrocardiographic monitoring [10]. The authors (using reference number 71 published in 2001) mentioned that "adverse outcome of either death or neurological sequalae was significantly less common in artemether group and treatment with arthemether was associated with significantly faster parasite clearance. In subgroup analyses, artemether was associated with a significantly lower mortality than quinine in adult with multisystem failure". However, WHO in 2010 [2] showed that "two systematic reviews and 3 subsequent randomized controlled (RCT) trials found no significant difference in death rates between the groups receiving artemether and quinine for severe malaria".

The authors [5] also mentioned the large RCT comparison of intravenous artesunate and quinine in 1461 patients "in Thailand" or SEAQUAMAT study. The study was actually multicenter study conducted in many countries "outside" Thailand (e.g. Bangladesh, India, Indonesia, and Myanmar) [12]

The authors mentioned [5] that maintenance dose of artesunate should be given for 3 days which was incorrect. Three days of intravenous artesunate administration alone is not enough for treatment of severe malaria. In 2010, WHO [2] recommended that parenteral antimalarials (including artesunate) should be given to treat severe malaria patients for a minimum of 24 hours, once started (irrespective of the patient's ability to tolerate oral medication earlier), and thereafter, complete the

*Corresponding author: Polrat Wilairatana, Faculty of Tropical Medicine, Department of Clinical Tropical Medicine, Mahidol University, 420/6 Rajvithi Road, Rajthevi, Bangkok 10400, Thailand, E-mail: polrat.wil@mahidol.ac.th

Received February 12, 2013; Accepted March 16, 2013; Published March 20, 2013

Citation: Wilairatana P, Tangpukdee N, Krudsood S (2013) Misconcepts in Management of Severe Malaria. Trop Med Surg 1: 111. doi:10.4172/ 2329-9088.1000111

Copyright: (c) 2013 Wilairatana P, et al. This is an open-access article distributed under the terms of the Creative Commons Attribution License, which permits unrestricted use, distribution, and reproduction in any medium, provided the original author and source are credited. 
Citation: Wilairatana P, Tangpukdee N, Krudsood S (2013) Misconcepts in Management of Severe Malaria. Trop Med Surg 1: 111. doi:10.4172/ 2329-9088.1000111

treatment by given a course of artemisin combination therapy (such as coartemether for 3 days or artesunate-doxycyclin/or clindamycin for 7 days).

Supportive Management [5]: The authors mentioned "Careful, serial monitoring of serum potassium with adequate supplement is suggested in patients with severe malaria complicated by acidosis". It unlikely occurs since potassium will be drived out of the cells into blood circulation during metabolic acidosis [13], therefore the risk of hyperkalemia will be higher in severe malaria patients than the risk of hypokalemia. Therefore potassium supplement in the patients with hyperkalemia may be harmful and may cause cardiac arrest. The clinicians should be aware of hyperkalemia rather than hypokalemia particularly if the severe malaria patients had concomitant metabolic acidosis and acute renal failure (as the severe malaria complications) during admission which may occur in some severe malaria patients. Although some studies indicated that hypokalemia was not apparent during admission but developed within 24 hours or during the correction of acidosis $[14,15]$.

In conclusion, clinicians who manage severe malaria should get updated information's otherwise misconcepted management may cause improper management and unfavorable outcome to the patients.

\section{References}

1. WHO (2006) Guidelines for the treatment of malaria ( $1^{\text {st }}$ edn), WHO, Geneva Switzerland. p 64-65.

2. WHO (2010) Guidelines for the treatment of malaria ( $2^{\text {nd }}$ edn), WHO, Geneva Switzerland. WHO.

3. WHO (2000) Management of severe: a practical handbook ( $2^{\text {nd }}$ edn), WHO, Geneva. Switzerland.

4. (2000) Severe falciparum malaria. World Health Organization, Communicable Diseases Cluster. Trans R Soc Trop Med Hyg 94: S1-90.
5. Sarkar PK, Ahluwalia G, Vijayan VK, Talwar A (2010) Critical care aspects of malaria. J Intensive Care Med 25: 93-103.

6. Looareesuwan S, Warrell DA, White NJ, Sutharasamai P, Chanthavanich P, et al. (1983) Do patients with cerebral malaria have cerebral oedema? A computed tomography study. Lancet 1: 434-437.

7. Looareesuwan S, Wilairatana P, Krishna S, Kendall B, Vannaphan S, et al (1995) Magnetic resonance imaging of the brain in patients with cerebral malaria. Clin Infect Dis 21: 300-309.

8. Patankar TF, Karnad DR, Shetty PG, Desai AP, Prasad SR (2002) Adult cerebral malaria: prognostic importance of imaging findings and correlation with postmortem findings. Radiology 224: 811-816.

9. Gupta S, Patel K (2008) Case series: MRI features in cerebral malaria. Indian J Radiol Imaging 18: 224-226.

10. White NJ (2009). Malaria. In: Cook GC, Zumla Al (eds). Manson's Tropical Diseases (22ndedn). Saunders Elsevier, China.

11. Warrell DA, Looareesuwan $S$, Warrell MJ, Kasemsarn $P$, Intaraprasert R, et al. (1982) Dexamethasone proves deleterious in cerebral malaria. A double-blind trial in 100 comatose patients. N Engl J Med 306: 313-319.

12. Dondorp A, Nosten F, Stepniewska K, Day N, White N; South East Asian Quinine Artesunate Malaria Trial (SEAQUAMAT) group (2005) Artesunate versus quinine for treatment of severe falciparum malaria: a randomised trial. Lancet 366: 717-725.

13. Dondorp AM, Fanello Cl, Hendriksen IC, Gomes E, Seni A, et al. (2010) Artesunate versus quinine in the treatment of severe falciparum malaria in African children (AQUAMAT): an open-label, randomised trial. Lancet 376 1647-1657.

14. Maitland K, Pamba A, Fegan G, Njuguna P, Nadel S, et al. (2005) Perturbations in electrolyte levels in kenyan children with severe malaria complicated by acidosis. Clin Infect Dis 40: 9-16.

15. Maitland K, Pamba A, Newton CR, Lowe B, Levin M (2004) Hypokalemia in children with severe falciparum malaria. Pediatr Crit Care Med 5: 81-85. 